\title{
Inhibition of autophagy by berberine enhances the survival of H9C2 myocytes following hypoxia
}

\author{
ZHUYIN JIA $^{1 *}$, LU LIN $^{2 *}$, SHANJUN HUANG $^{2}$, ZHOUYANG ZHU $^{2}$, WEIJIAN HUANG ${ }^{2}$ and ZHOUQING HUANG ${ }^{2}$ \\ ${ }^{1}$ Department of Cardiology, Wenzhou Central Hospital; ${ }^{2}$ Cardiac Center, \\ Department of Cardiology, The Key Laboratory of Cardiovascular Disease of Wenzhou, \\ The First Affiliated Hospital of Wenzhou Medical University, Wenzhou, Zhejiang 325000, P.R. China
}

Received February 13, 2016; Accepted February 21, 2017

DOI: $10.3892 / \mathrm{mmr} .2017 .6770$

\begin{abstract}
Hypoxia may induce apoptosis and autophagy to promote cardiomyocyte injury. The present study investigated the effect of berberine, a natural extract of Rhizoma Coptidis, on hypoxia-induced autophagy and apoptosis in the H9c2 rat myocardial cell line. Expression levels of apoptosis and autophagy markers were upregulated in $\mathrm{H} 9 \mathrm{c} 2$ myocytes during hypoxia and cell viability was reduced. However, berberine significantly reduced hypoxia-induced autophagy in $\mathrm{H} 9 \mathrm{c} 2$ myocytes, as demonstrated by the ratio of microtubule-associated proteins 1A/1B light chain $3 \mathrm{I} / \mathrm{II}$ and the expression levels of B-cell lymphoma 2 (Bcl-2)/adenovirus E1B $19 \mathrm{kDa}$ protein-interacting protein 3 , and promoted cell viability. In addition, expression levels of the Bcl-2 anti-apoptotic protein were significantly downregulated, and expression levels of pro-apoptotic proteins Bcl-2-associated X protein and cleaved caspase-3 were upregulated during hypoxia injury in cardiac myocytes. This was reversed by treatment with berberine or the autophagy inhibitor 3-methyladenine, whereas the autophagy agonist rapamycin had the opposite effects, suggesting that berberine reduces myocyte cell death via inhibition of autophagy and apoptosis during hypoxia. In addition, Compound $\mathrm{C}$, a 5 ' adenosine monophosphate-activated protein kinase (AMPK) inhibitor, reduced apoptosis and autophagy in hypoxic myocytes, suggesting that the activation of the AMPK signaling pathway may be involved in this process. These findings suggested that berberine protects cells from hypoxia-induced apoptosis via inhibition of autophagy and suppression of AMPK activation. Therefore, berberine may be
\end{abstract}

Correspondence to: Dr Zhouqing Huang, Cardiac Center, Department of Cardiology, The Key Laboratory of Cardiovascular Disease of Wenzhou, The First Affiliated Hospital of Wenzhou Medical University, 2 Fuxue Road, Wenzhou, Zhejiang 325000, P.R. China

E-mail: susiehzq@126.com

*Contributed equally

Key words: berberine, autophagy, apoptosis, hypoxia, myocytes a potential therapeutic agent for the treatment of patients with cardiac myocyte injury and ischemia.

\section{Introduction}

Ischemic heart disease is a pervasive health problem worldwide. Severe ischemia or hypoxia induces activation of cell death mechanisms including necrosis, apoptosis and autophagy. Autophagy is an important process for the degradation and recycling of long-lived proteins and cytoplasmic organelles, and is activated in response to a variety of extracellular and intracellular stimuli (1). Therefore, autophagy serves a cell protective role under certain circumstances. For example, transient and moderate ischemia induced autophagy via a 5' adenosine monophosphate-activated protein kinase (AMPK)-dependent mechanism. Glucose deprivation (GD), which mimics myocardial ischemia, induced autophagy in cultured cardiac myocytes. Survival of cardiac myocytes was decreased by 3-methyladenine, an inhibitor of autophagy, suggesting that autophagy is protective against GD in cardiac myocytes. GD-induced autophagy coincided with activation of AMPK (2). However, autophagy induced by acute ischemia is associated with reduced cell viability, possibly due to the non-specific degradation of cytoplasmic contents $(3,4)$. Therefore, inhibition of autophagy during severe hypoxia may be beneficial for the survival of myocardial cells and may reduce cardiac injury.

Autophagy and apoptosis serve essential roles in cell death and regulate cardiovascular disease. Studies have demonstrated that following cardiac ischemia/reperfusion injury, autophagy and apoptosis are stimulated to reduce cell survival via activation of the mitochondrial c-Jun N-terminal kinase signaling pathway (5-7). However, the association between autophagy and apoptosis is complex. An early study revealed that in response to stress stimuli, autophagy may trigger apoptosis and lead to cell death (8). In addition, the two pathways share common regulatory factors and each may influence and alter the activity of the other (9). Therefore, it is important to investigate the interaction between autophagy and apoptosis during hypoxia in the heart, as the levels of the two processes must be regulated to protect cells.

Berberine, an isoquinoline alkaloid, is derived from herbs, including Hydrastis canadensis (goldenseal) and 
Coptischinensis(Coptisorgoldenthread)fromtheRanunculaceae family, Arcangelisia flava from the Menispermaceae family, and Berberis aquifolium (Oregon grape) and Berberis aristata (tree turmeric) from the Berberidaceae family $(10,11)$. Berberine may have the potential to treat a wide range of diseases, including endothelial dysfunction, hyperlipemia and diabetes (12-14). In addition, our previous study demonstrated that berberine may significantly decrease infarct size and improve cardiac function following ischemia/reperfusion-induced myocardial injury, and this may involve the inhibition of excessive autophagy (15). Various studies have demonstrated that berberine may protect against apoptosis following pathological ischemia (16-18). However, the effect of berberine on autophagy in myocardial cells during hypoxia remains to be determined. The present study demonstrated that berberine treatment significantly improved cell viability and suppressed autophagy and apoptosis in hypoxia-induced myocardial cells. Additionally, the underlying mechanism of berberine protection was investigated.

\section{Materials and methods}

Reagents. Dulbecco's modified Eagle's medium (DMEM), fetal bovine serum (FBS) and penicillin/streptomycin (pen/strep; $10,000 \mathrm{U} / \mathrm{ml}$ each) were purchased from Gibco; Thermo Fisher Scientific, Inc. (Waltham, MA, USA). Dimethyl sulfoxide (DMSO) and berberine were obtained from Sigma-Aldrich; Merck KGaA (Darmstadt, Germany). A rabbit antibody against $\beta$-actin (catalog no. ab8227) was obtained from Abcam (Cambridge, UK). Rabbit anti-microtubule-associated proteins 1A/1B light chain 3 (LC3) B (catalog no. 2775), rabbit anti-B-cell lymphoma 2 (Bcl-2)/adenovirus E1B $19 \mathrm{kDa}$ protein-interacting protein 3 (BNIP3; catalog no. 12396), rabbit anti-Bcl-2 (catalog no. 2870), rabbit anti-caspase-3 (catalog no. 9665) and rabbit anti-Bcl-2-associated X protein (Bax; catalog no. 2772) antibodies were purchased from Cell Signaling Technology, Inc. (Danvers, MA, USA). A goat anti-rabbit secondary antibody conjugated to Alexa Fluor ${ }^{\circledR} 680$ (catalog no. A-21109) was obtained from Invitrogen; Thermo Fisher Scientific, Inc.

Cell culture and treatment. The $\mathrm{H} 9 \mathrm{c} 2$ rat myocardium-derived cell line was purchased from the American Type Culture Collection (Manassas, VA, USA). Cells were cultured in DMEM containing 4,500 $\mathrm{mg} / 1$ glucose and supplemented with 10\% (v/v) FBS, 10 mM 4-(2-hydroxyethyl)-1-piperazinethanesulfonic acid (Sigma Aldrich; Merck KGaA) and $1 \%$ pen/strep solution at $37^{\circ} \mathrm{C}$ in a $5 \% \mathrm{CO}_{2}$ incubator. To determine the optimal duration of hypoxia, cells at $\sim 90 \%$ confluence were resuspended in serum-free and low glucose $(1,500 \mathrm{mg} / \mathrm{l})$ DMEM and incubated for $4 \mathrm{~h}$. Subsequently, cells were cultured under hypoxic conditions $\left(1 \% \mathrm{O}_{2}, 5 \% \mathrm{CO}_{2}\right.$ and $94 \% \mathrm{~N}_{2}$ ) for 1 to $12 \mathrm{~h}$. Berberine (Sigma Aldrich; Merck $\mathrm{KGaA} ; 5,10$ or $25 \mu \mathrm{M}$ ) dissolved in DMSO, $5 \mathrm{mM}$ 3-methyladenine (Sigma Aldrich; Merck KGaA) dissolved in PBS or $10 \mathrm{nM}$ rapamycin (Cell Signaling Technology, Inc., Danvers, MA, USA) dissolved in PBS, $10 \mu \mathrm{M}$ Compound C (Sigma Aldrich; Merck KGaA) dissolved in DMSO, were added to cells, which were seeded in 6 -well plates at $1 \times 10^{6}$ cells/well, following a 4-h incubation in serum-free low glucose DMEM, and incubated under normoxic conditions $\left(21 \% \mathrm{O}_{2}, 5 \% \mathrm{CO}_{2}\right.$ and $74 \% \mathrm{~N}_{2}$ ) for $1 \mathrm{~h}$ prior to culturing under hypoxic conditions $\left(1 \% \mathrm{O}_{2}, 5 \% \mathrm{CO}_{2}\right.$ and $\left.94 \% \mathrm{~N}_{2}\right)$ for $6 \mathrm{~h}$. For the control group, cells were cultured constantly under normoxic condition.

Determination of cell viability. The 3-(4,5-dimethylthiazol-2-yl)-2,5-diphenyltetrazolium bromide (MTT) assay was utilized to assess cell viability. Cells were seeded in 96-well culture plates $\left(3 \times 10^{3}\right.$ per well). Following treatment, cells were incubated with $0.5 \mathrm{mg} / \mathrm{ml}$ MTT (Roche Applied Science, Penzberg, Germany) in DMEM for $4 \mathrm{~h}$. The blue formazan crystals produced from viable cells were dissolved in DMSO. Absorbance was measured spectrophotometrically at a wavelength of $570 \mathrm{~nm}$.

Protein isolation and western blot analysis. Following treatment, cells were washed with cold PBS ( $\mathrm{pH} 7.4)$ and lysed for $30 \mathrm{~min}$ with lysis buffer [0.5\% Nonidet $40,50 \mathrm{mmol} / 1$ Tris-HCl (pH 7.5), $1 \mathrm{mmol} / 1$ EDTA, $1 \mathrm{mmol} / 1$ EGTA and $150 \mathrm{mmol} / \mathrm{l} \mathrm{NaCl}$, containing $10 \%$ glycerol, $50 \mathrm{mmol} / \mathrm{l}$ sodium fluoride, $10 \mathrm{mmol} / 1$ sodiumpyrophosphate, $1 \mathrm{mmol} / 1$ sodium orthovanadate, $80 \mu \mathrm{mol} / 1 \beta$-glycerophosphate, $1 \mathrm{mmol} / 1$ phenylmethanesulfonyl fluoride, $10 \mu \mathrm{g} / \mathrm{ml}$ aprotinin, $100 \mu \mathrm{g} / \mathrm{ml}$ soybean trypsin inhibitor and $10 \mu \mathrm{g} / \mathrm{ml}$ leupeptin] and centrifuged for $10 \mathrm{~min}$ at $12,000 \mathrm{x} \mathrm{g}$ at $4^{\circ} \mathrm{C}$. Subsequently, protein concentrations were measured using a Bicinchoninic Acid assay (Pierce; Thermo Fisher Scientific, Inc.). Cell lysates $(20 \mu \mathrm{g})$ were loaded onto $10 \%$ polyacrylamide SDS gels and subjected to electrophoresis. Proteins were subsequently transferred onto polyvinylidene difluoride membranes (EMD Millipore, Billerica, MA, USA). Membranes were blocked with $5 \%$ skimmed milk in TBS containing $0.05 \%$ Tween-20 (TBST) for $1 \mathrm{~h}$ at room temperature, and probed with anti-LC3B, anti-Bcl-2, anti-Bax, anti-cleaved caspase-3, anti-BNIP3 (diluted 1:1,000 in TBST) or anti- $\beta$-actin (diluted 1:5,000 in TBST) primary antibodies for $24 \mathrm{~h}$ at $4{ }^{\circ} \mathrm{C}$, followed by a goat anti-rabbit secondary antibody (diluted 1:1,000 in TBST) for $1 \mathrm{~h}$ at $25^{\circ} \mathrm{C}$, conjugated to the far-red-fluorescent Alexa Fluor 680 dye. Signals were detected by the Odyssey ${ }^{\circledR}$ Imaging system (LI-COR Biosciences, Lincoln, NE, USA). Densitometric analysis was performed using Quantity One software version 4.6.2 (Bio-Rad Laboratories, Inc., Hercules, CA, USA).

Annexin V/propidium iodide (PI) double staining and flow cytometry. Apoptosis was measured by flow cytometry, which was performed using Annexin V, FITC Apoptosis Detection kit (Dojindo Molecular Technologies, Inc., Kumamoto, Japan) according to the manufacturer's protocol. Cells seeded at $1 \times 10^{6}$, were incubated with $5 \mu \mathrm{l}$ Annexin V-FITC and $5 \mu \mathrm{l}$ PI at room temperature for $15 \mathrm{~min}$ in the dark and apoptotic cells were detected by flow cytometry. The mean fluorescence intensity of Annexin V/PI staining in the myocytes was analyzed using the BD FACSCalibur ${ }^{\mathrm{TM}}$ (BD Biosciences, Franklin Lakes, NJ, USA) and FlowJo software version 7.61 (Stanford University, CA, USA) was used to analyze the results.

Statistical analysis. Three or more groups were compared by one-way analysis of variance followed by the Student-Newman-Keuls and Dunnett post hoc tests. Data are expressed as the mean \pm standard deviation and were analyzed using SPSS software version 18.0 (SPSS, Inc., Chicago, IL, 
A

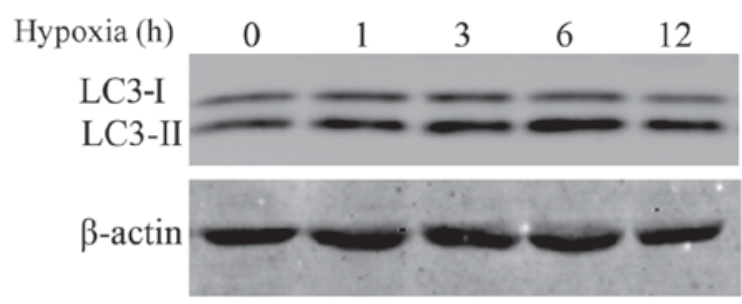

B

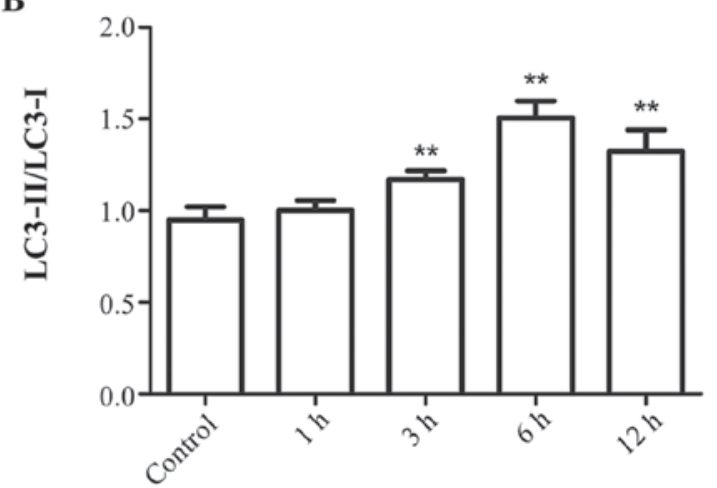

Figure 1. Expression levels of autophagy-associated proteins following hypoxia in H9c2 myocytes. Following 0-12 h hypoxia, whole cell lysates were collected for western blot analysis. (A) Representative blots of LC3-I, LC3-II and $\beta$-actin. (B) Densitometry of LC3-II/LC3-I signals. The protein expression level of the control $(0 \mathrm{~h})$ group was arbitrarily set as 1 in each blot, and the target protein signals on the same blot were normalized to the control to generate relative densities. Data are expressed as the mean \pm standard deviation and were obtained from three independent experiments. ${ }^{* *} \mathrm{P}<0.01$ vs. control. LC3; microtubule-associated proteins $1 \mathrm{~A} / 1 \mathrm{~B}$ light chain 3.

USA). $\mathrm{P}<0.05$ was considered to indicate a statistically significant difference. Experiments were performed at least three times.

\section{Results}

Effect of hypoxia duration on autophagy in myocytes. H9c2 myocytes were exposed to hypoxic conditions for 1 to $12 \mathrm{~h}$. Subsequently, protein expression levels of LC3-I and LC3-II were measured by western blotting. The greatest increase in the ratio of LC3-II/LC3-I expression levels compared with the control group was observed in cells subjected to hypoxia for $6 \mathrm{~h}$. Therefore the following experiments were all performed with cells subjected to hypoxia for $6 \mathrm{~h}$ (Fig. 1).

Berberine attenuates hypoxia-induced autophagy and apoptosis in H9c2 myocytes. The effect of berberine on autophagy and apoptosis in hypoxic myocytes was investigated. H9c2 cells were pretreated with 5-25 $\mu \mathrm{M}$ berberine for $1 \mathrm{~h}$. Hypoxia exposure reduced cell viability compared with the control group; this effect was abrogated by berberine treatment in dose-dependent manner, as determined by an MTT assay (Fig. 2A). In addition, there was a marked increase in apoptosis following hypoxia, as determined by the increased percentage of Annexin V/PI double positive cells (Fig. 2B), upregulation of the pro-apoptotic proteins Bax and caspase-3 (Fig. 3). A similar effect on autophagy was observed, which was demonstrated by the ratio of LC3-II/LC3-I and BNIP3 protein expression levels (Fig. 3). Treatment with berberine reversed this effect in a dose-dependent manner. This data suggested that berberine enhanced cell survival via inhibition of proteins involved in autophagy and apoptosis.

Inhibition of autophagy reduces apoptosis in hypoxic $\mathrm{H} 9 \mathrm{c} 2$ cells. As berberine significantly blocked apoptosis and the expression levels of autophagy-associated proteins in hypoxic H9c2 cells, the association between autophagy and apoptosis was investigated using the autophagy inhibitor 3-MA and the autophagy inducer rapamycin. Hypoxia treatment markedly reduced H9c2 cell viability (Fig. 4A) and enhanced apoptosis
(Fig. 4B) compared with the control. However, in the presence of 3-MA, cell viability was enhanced and apoptosis was reduced compared with the hypoxia group, whereas rapamycin treatment had the opposite effect (Fig. 4). In addition, similar results were observed in protein expression levels as measured by western blotting. Expression levels of the autophagy markers LC3-II and BNIP3, the pro-apoptosis proteins Bax and cleaved caspase-3 were significantly reduced by $3-\mathrm{MA}$ treatment $(\mathrm{P}<0.01)$, whereas the anti-apoptosis protein Bcl-2 was significantly enhanced $(\mathrm{P}<0.01$; Fig. 5), compared with the hypoxic group. However, rapamycin treatment significantly increased the expression levels of LC3-II, BNIP3 and Bax, and reduced Bcl-2 expression compared with the control group ( $\mathrm{P}<0.01$; Fig. 5). These results suggested that inhibition of autophagy reduced apoptosis and increased cell viability in hypoxic myocytes.

AMPK signaling pathway activation may be involved in the regulation of apoptosis by berberine in hypoxic $\mathrm{H} 9 \mathrm{c} 2$ cells. To confirm the effect of berberine on the inhibition of apoptosis, the specific AMPK inhibitor, Compound C, was utilized. Compound $\mathrm{C}$ treatment significantly reduced cellular apoptosis during hypoxia, compared with hypoxia treatment alone. The reduction in apoptosis by Compound $\mathrm{C}$ was comparable to that of berberine treatment or berberine and Compound $\mathrm{C}$ in combination, suggesting that AMPK activation may be involved in the regulation of apoptosis in hypoxic $\mathrm{H} 9 \mathrm{c} 2$ cells, and the inhibitory effect of berberine on apoptosis may be associated with the inhibition of AMPK activation.

\section{Discussion}

The present study demonstrated that berberine effectively reduced the levels of apoptosis and the protein expression levels of autophagy markers, and enhanced cell survival in hypoxic myocardial cells. In addition, the inhibition of autophagy reduced apoptosis and further improved cell viability. These observations suggested that berberine increases cell survival by decreasing autophagy to reduce cell apoptosis in hypoxic myocytes. Furthermore, inhibition of AMPK activation may be involved in the regulation of apoptosis by berberine. 
A
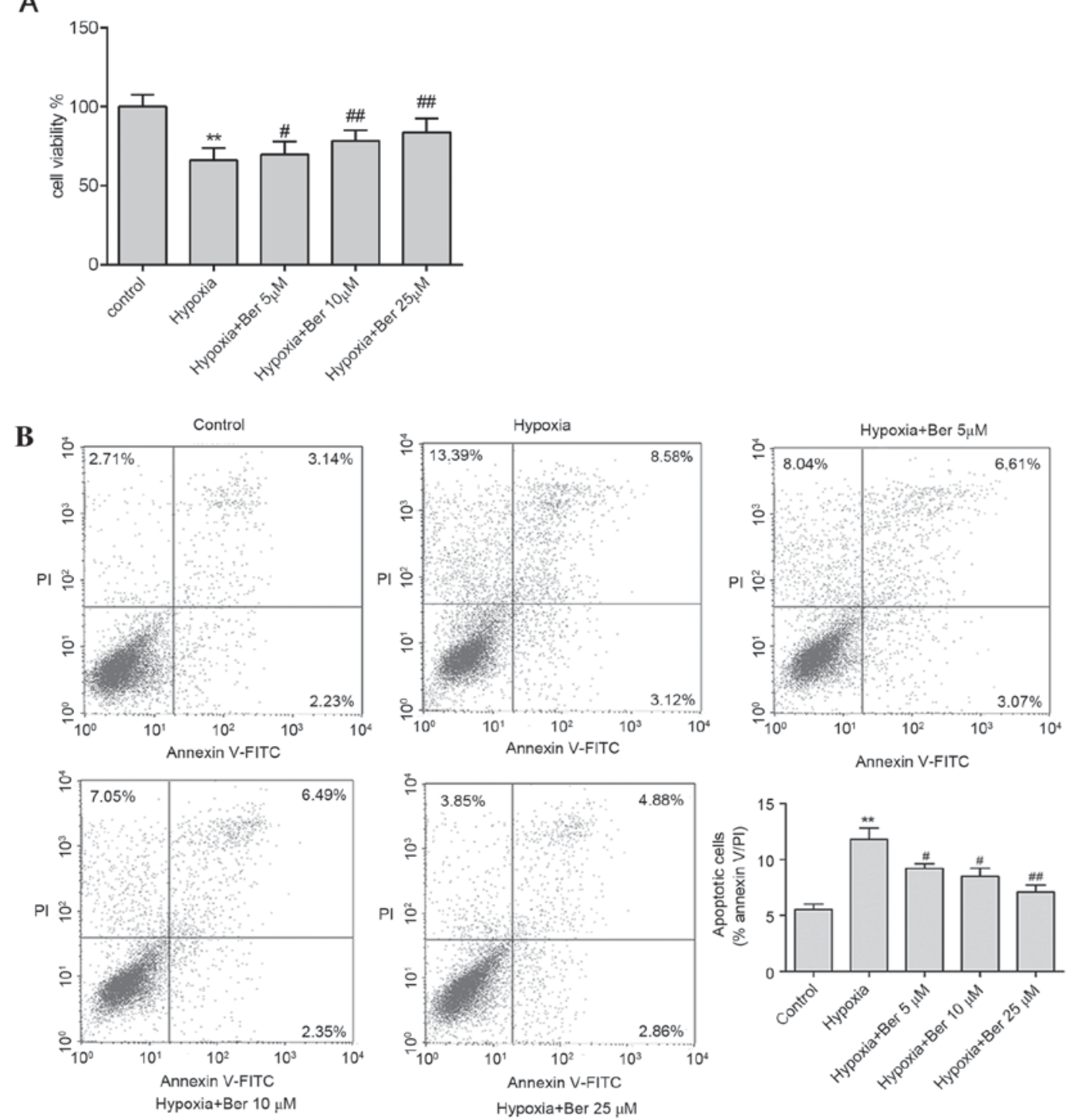

Figure 2. Effect of berberine on cell viability and apoptosis in $\mathrm{H} 9 \mathrm{c} 2$ cells exposed to hypoxia. Cells were treated with $0,5,10$ or $25 \mu \mathrm{M}$ berberine, prior to hypoxia. (A) Cell viability of $\mathrm{H} 9 \mathrm{c} 2$ cells, as assessed by an MTT assay. (B) Induction of apoptosis in H9c2 cells was measured by Annexin-V/PI double-staining followed by flow cytometric analysis. The upper right and lower right areas, which represented the percentage of late apoptosis and early apoptosis, were analyzed. Data are expressed as the mean \pm standard deviation and were obtained from three independent experiments. ${ }^{* *} \mathrm{P}<0.01$ vs. control group; ${ }^{*} \mathrm{P}<0.05$ and ${ }^{\# \#} \mathrm{P}<0.01$ vs. hypoxia group. Ber, berberine; FITC, fluorescein isothiocyanate; PI, propidium iodide.

Autophagy is involved in various physiological and pathological cellular processes, including development, differentiation, inflammation, immunity, metabolism and cell death (19-21). Studies have suggested that autophagy serves a protective role in cells during ischemia. For example, Yan et al (22) demonstrated that autophagy may be activated by repetitive myoca+rdial ischemia in pigs, and may serve as a homeostatic mechanism to suppress apoptosis and to protect myocardium from the deleterious effects of chronic ischemia. In addition, the protective effect of autophagy was demonstrated in models of brain (23) and renal (24) ischemia. Studies by Hamacher-Brady et al (25) and Dosenko et al (26) supported these findings, which suggested that regulated autophagy serves a role in cell survival. However, autophagy is a double-edged sword that mediates cell death under specific circumstances (9). Excessive autophagy leads to cell death via destruction of the cytosol and organelles (27). Evidence suggests that an uncontrolled and excessive induction of autophagy during reperfusion may lead to cellular dysfunction in cardiomyocytes, and eventually to autophagic cell death $(2,3,28)$. The present study investigated the effect of autophagy during hypoxia in $\mathrm{H} 9 \mathrm{c} 2$ cells. The maximum level of autophagy was observed following $6 \mathrm{~h}$ of hypoxia, and the inhibition of autophagy by 3 -MA markedly improved cell viability. This data suggested that the reduction of autophagy may benefit cell survival in certain circumstances, and that autophagy may be detrimental to the cell during hypoxia. Notably, Aki et al (29) reported similar results following glucose starvation.

There is considerable crosstalk between autophagy and apoptosis pathways. One molecule that may connect autophagy and apoptosis is BNIP3, which is a BH3-domain-containing member of the mitochondrial pro-apoptotic Bcl-2 family (30). It 

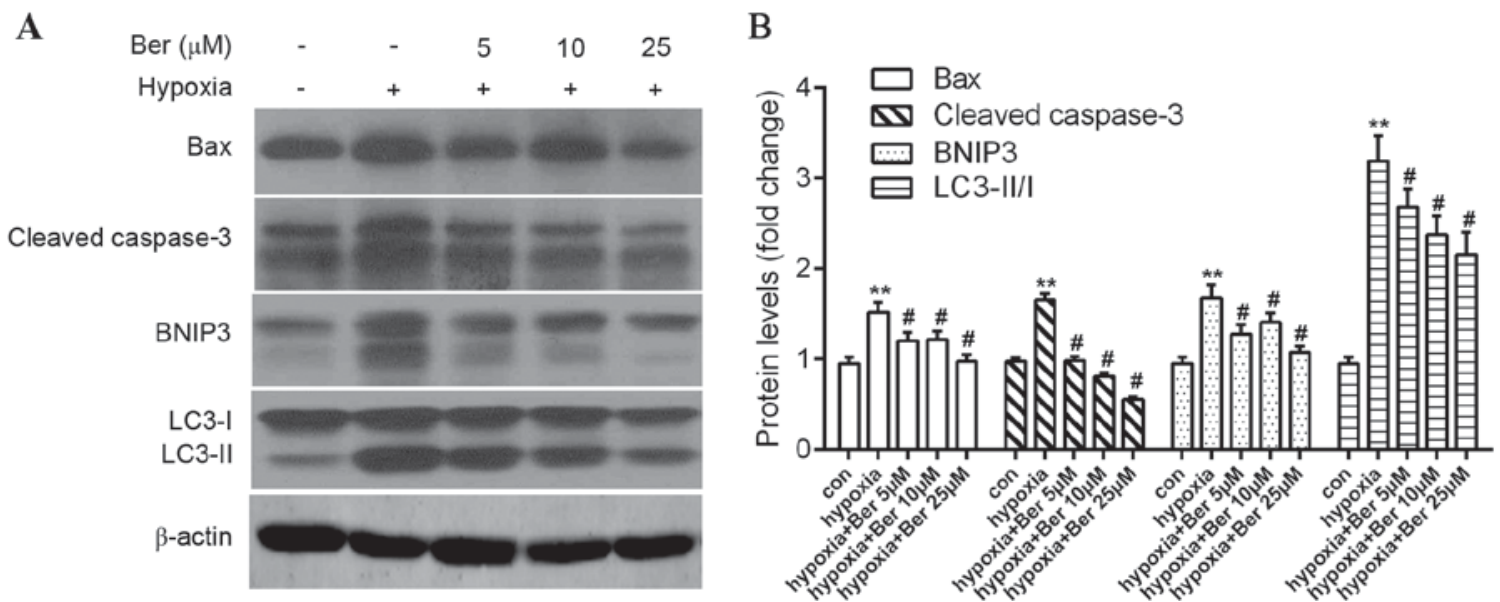

Figure 3. Berberine inhibits the hypoxia-induced increase in the expression levels of autophagy- and apoptotic-associated proteins. (A) Protein expression levels of Bax, cleaved caspase-3, BNIP3, LC3 and $\beta$-actin were measured by western blot analysis. (B) Quantification of bands by densitometry. $\beta$-actin served as the loading control. Data are expressed as the mean \pm standard deviation and were obtained from three independent experiments. " $\mathrm{P}<0.01$ vs. control group; ${ }^{\text {"}} \mathrm{P}<0.01$ vs. hypoxia group. Bax, B-cell lymphoma 2-associated X protein; BNIP3, B-cell lymphoma 2/adenovirus E1B 19 kDa protein-interacting protein 3; LC3, microtubule-associated proteins 1A/1B light chain 3; Ber, berberine.

A
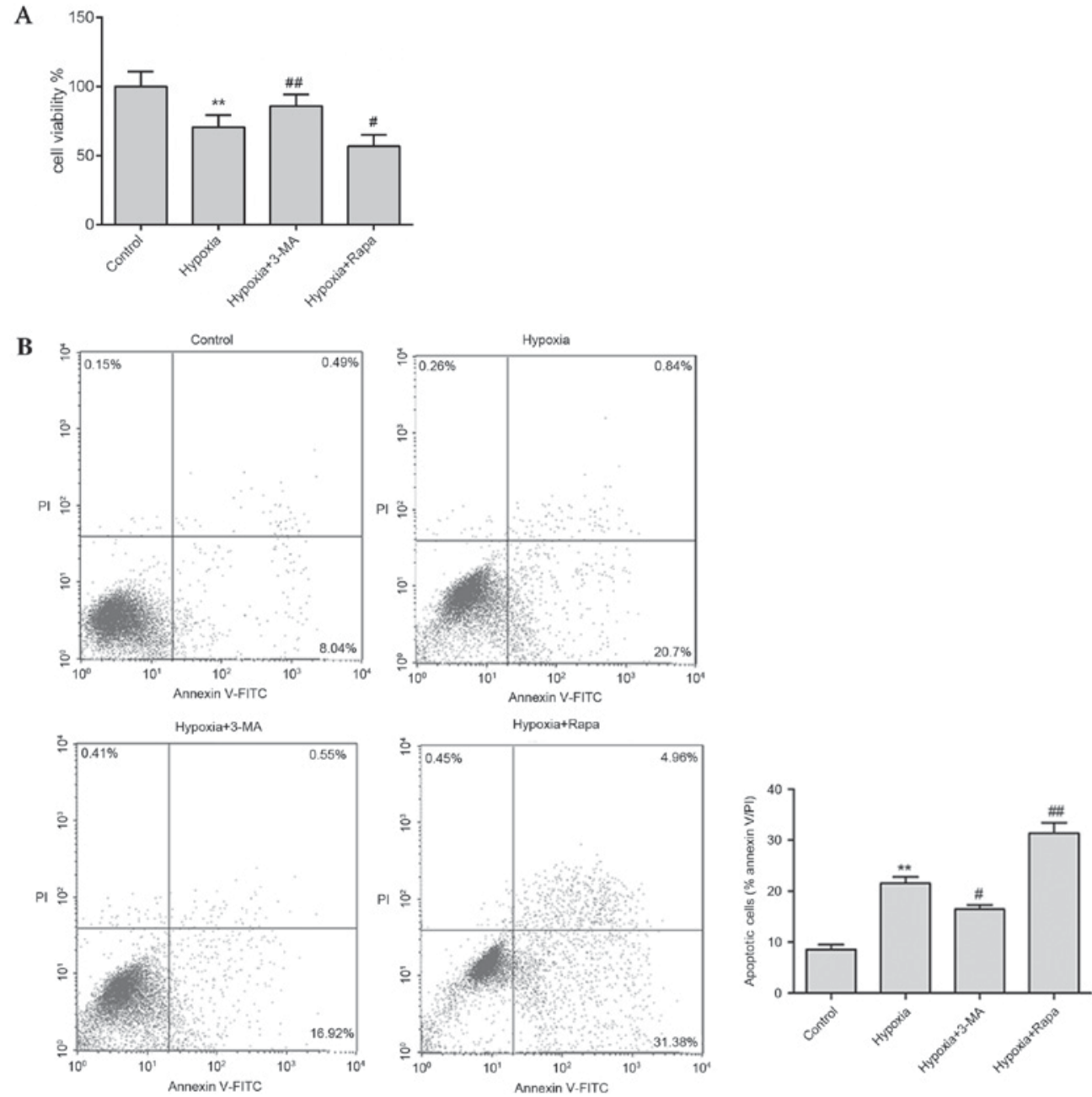

Figure 4. Effect of 3-MA or rapamycin on cell viability and apoptosis in hypoxic H9c2 cells. Cells were subjected to glucose starvation with the exception of the control group, which was cultured in complete Dulbecco's modified Eagle's medium. Subsequently, cells were treated with 3-MA (an autophagy inhibitor) or rapamycin (an autophagy inducer) prior to hypoxia exposure. (A) Viability of H9c2 cells. (B) Induction of apoptosis in H9c2 cells was measured by Annexin-V/PI double-staining followed by flow cytometric analysis. The apoptotic proportion of H9c2 cells was calculated according to the flow cytometry results. The upper right and lower right areas, which represented the percentage of late apoptosis and early apoptosis, were analyzed. Data are expressed as the mean \pm standard deviation and were obtained from three independent experiments. ${ }^{* *} \mathrm{P}<0.01$ vs. control group; ${ }^{*} \mathrm{P}<0.05$ and ${ }^{\# \#} \mathrm{P}<0.01$ vs. hypoxia group. $3-\mathrm{MA}$, 3-methyladenine; FITC, fluorescein isothiocyanate; PI, propidium iodide; Rapa, rapamycin. 

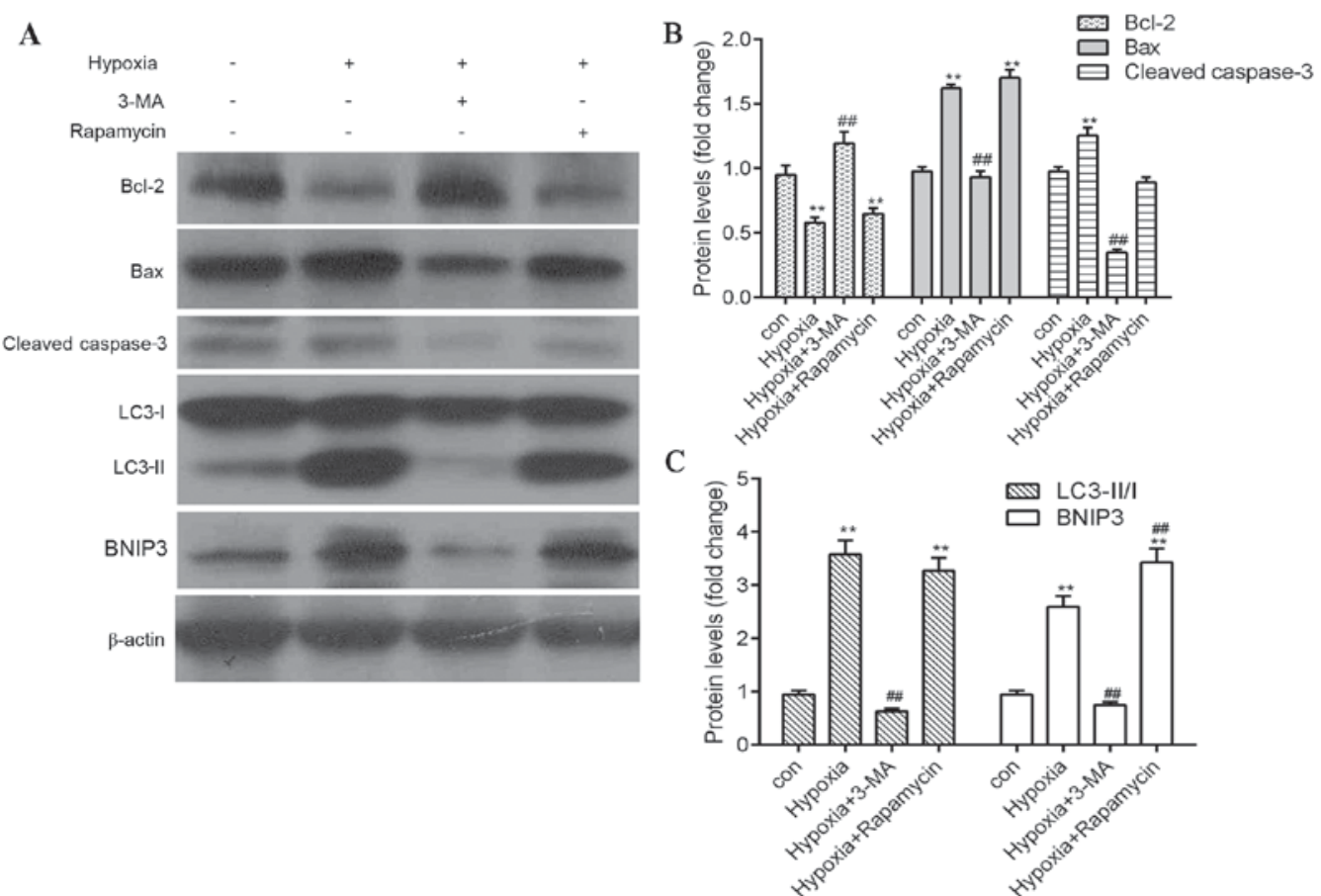

Figure 5. Inhibition of autophagy reduces apoptosis in hypoxia-induced myocytes. (A) Protein expression levels of Bax, Bcl-2, cleaved caspase-3, BNIP3, LC3 and $\beta$-actin were measured by western blot analysis. Quantification of (B) Bcl-2, Bax and cleaved caspase-3, and (C) LC3-II/LC3-I and BNIP3 protein expression levels by densitometry. $\beta$-actin served as the loading control. Data are expressed as the mean \pm standard deviation and were obtained from three independent experiments. ${ }^{* *} \mathrm{P}<0.01$ vs. control group; ${ }^{\# \#} \mathrm{P}<0.01$ vs. hypoxia group. Bax, B-cell lymphoma 2-associated $\mathrm{X}$ protein; Bcl-2, B-cell lymphoma 2; BNIP3, B-cell lymphoma 2/adenovirus E1B 19 kDa protein-interacting protein 3; LC3, microtubule-associated proteins 1A/1B light chain 3; 3-MA, 3-methyladenine.
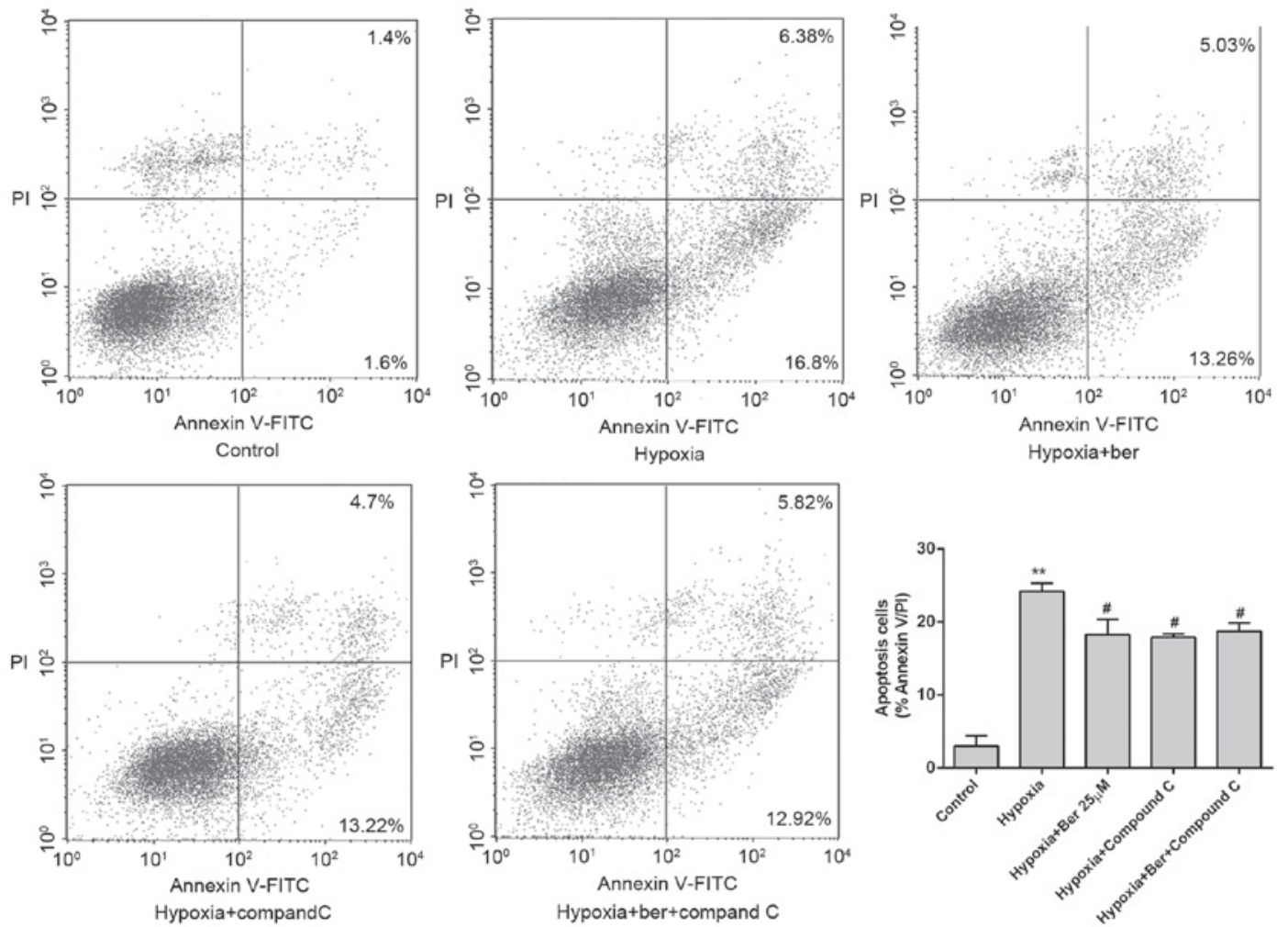

Figure 6. Activation of the AMPK signaling pathway may be involved in the regulation of apoptosis by berberine in hypoxic H9c2 cells. Cells were subjected to glucose starvation with the exception of the control group, which was cultured in complete Dulbecco's modified Eagle's medium. Cells were treated with berberine, Compound C (an AMPK inhibitor) or a combination of the two, prior to hypoxia exposure. Induction of apoptosis in H9c2 cells was measured by Annexin-V/PI double-staining followed by flow cytometric analysis. The apoptotic proportion of H9c2 cells was calculated according to the flow cytometry results. The upper right and lower right areas, which represented the percentage of late apoptosis and early apoptosis, were analyzed. Data are expressed as the mean \pm standard deviation and were obtained from three independent experiments. ${ }^{* *} \mathrm{P}<0.01$ vs. control group; ${ }^{*} \mathrm{P}<0.05$ vs. hypoxia group. AMPK, $5^{\prime}$ adenosine monophosphate-activated protein kinase; FITC, fluorescein isothiocyanate; PI, propidium iodide; Ber, berberine. 
has been reported that in hypoxia-induced neonatal myocytes, upregulated expression of BNIP3 resulted in mitochondrial dysfunction and subsequent apoptosis (31-33). Wang et al (34) demonstrated that during ischemia, BNIP3 was highly upregulated, which subsequently stimulated mitochondrial perturbations, autophagy and cell death. In the present study, hypoxic H9c2 cells pretreated with 3-MA expressed significantly decreased levels of BNIP3, and reduced levels of the pro-apoptotic proteins Bax and caspase-3. Therefore, these findings suggested that inhibition of autophagy may reduce cellular apoptosis and enhance cell survival in hypoxic H9c2 cells.

Our previous study revealed that berberine significantly alleviated reperfusion injury, and enhanced the survival of myocytes via inhibition of excessive autophagy. However, it is unclear what role berberine serves during hypoxia in myocardial cells. The findings of the present study revealed that berberine markedly inhibited the ratio of LC3-II/LC3-I and the protein expression levels of BNIP3, Bax and cleaved caspase-3. This suggested that berberine attenuates cell injury during hypoxia via inhibition of autophagy and apoptosis in H9c2 cells. In conclusion, the results of the present study suggested that berberine treatment protects against hypoxia-induced myocardial injury by selectively inhibiting excessive autophagy and reducing cell apoptosis. Given that cells pre-treated with berberine prior to hypoxia could suppress excessive autophagy, berberine may be a potential therapeutic agent for preventing myocardial ischemia injury, but further in vivo studies are required.

\section{Acknowledgements}

The present study was supported by the Traditional Chinese Medicine Administration of Zhejiang Province (grant no. 2016ZA137) and Wenzhou Science \& Technology Bureau (grant nos. Y20150036 and Y20150035).

\section{References}

1. Kelekar A: Autophagy. Ann N Y Acad Sci 1066: 259-271, 2005.

2. Matsui Y, Takagi H, Qu X, Abdellatif M, Sakoda H, Asano T, Levine B and Sadoshima J: Distinct roles of autophagy in the heart during ischemia and reperfusion: Roles of AMP-activated protein kinase and Beclin 1 in mediating autophagy. Circ Res 100: 914-922, 2007.

3. Valentim L, Laurence KM, Townsend PA, Carroll CJ, Soond S, Scarabelli TM, Knight RA, Latchman DS and Stephanou A: Urocortin inhibits Beclin1-mediated autophagic cell death in cardiac myocytes exposed to ischaemia/reperfusion injury. J Mol Cell Cardiol 40: 846-852, 2006.

4. Mizushima N, Levine B, Cuervo AM and Klionsky DJ: Autophagy fights disease through cellular self-digestion. Nature 451: 1069-1075, 2008.

5. Xu J, Qin X, Cai X, Yang L, Xing Y, Li J, Zhang L, Tang Y, Liu J, Zhang $X$ and Gao F: Mitochondrial JNK activation triggers autophagy and apoptosis and aggravates myocardial injury following ischemia/reperfusion. Biochim Biophys Acta 1852: 262-270, 2015

6. Ferrandi C, Ballerio R, Gaillard P, Giachetti C, Carboni S, Vitte PA, Gotteland JP and Cirillo R: Inhibition of c-Jun $\mathrm{N}$-terminal kinase decreases cardiomyocyte apoptosis and infarct size after myocardial ischemia and reperfusion in anaesthetized rats. Br J Pharmacol 142: 953-960, 2004.

7. Chambers JW, Pachori A, Howard S, Iqbal S and LoGrasso PV: Inhibition of JNK mitochondrial localization and signaling is protective against ischemia/reperfusion injury in rats. J Biol Chem 288: 4000-4011, 2013.
8. Espert L, Denizot M, Grimaldi M, Robert-Hebmann V, Gay B, Varbanov M, Codogno P and Biard-Piechaczyk M: Autophagy is involved in T cell death after binding of HIV-1 envelope proteins to CXCR4. J Clin Invest 116: 2161-2172, 2006.

9. Maiuri MC, Zalckvar E, Kimchi A and Kroemer G: Self-eating and self-killing: Crosstalk between autophagy and apoptosis. Nat Rev Mol Cell Biol 8: 741-752, 2007.

10. Peng WH, Wu CR, Chen CS, Chen CF, Leu ZC and Hsieh MT: Anxiolytic effect of berberine on exploratory activity of the mouse in two experimental anxiety models: Interaction with drugs acting at 5-HT receptors. Life Sci 75: 2451-2462, 2004

11. Wang F, Zhao G, Cheng L, Zhou HY, Fu LY and Yao WX: Effects of berberine on potassium currents in acutely isolated CA1 pyramidal neurons of rat hippocampus. Brain Res 999: 91-97, 2004.

12. Zhang M, Wang CM, Li J, Meng ZJ, Wei SN, Li J, Bucala R, $\mathrm{Li}$ YL and Chen L: Berberine protects against palmitate-induced endothelial dysfunction: Involvements of upregulation of AMPK and eNOS and downregulation of NOX4. Mediators Inflamm 2013: 260464, 2013.

13. Heidarian E, Rafieian-Kopaei M, Khoshdel A and Bakhshesh M: Metabolic effects of berberine on liver phosphatidate phosphohydrolase in rats fed on high lipogenic diet: An additional mechanism for the hypolipidemic effects of berberine. Asian Pac J Trop Biomed 4 (Suppl 1): S429-S435, 2014.

14. Vuddanda PR, Chakraborty S and Singh S: Berberine: A potential phytochemical with multispectrum therapeutic activities. Expert Opin Investig Drugs 19: 1297-1307, 2010.

15. Huang Z, Han Z, Ye B, Dai Z, Shan P, Lu Z, Dai K, Wang C and Huang W: Berberine alleviates cardiac ischemia/reperfusion injury by inhibiting excessive autophagy in cardiomyocytes. Eur J Pharmacol 762: 1-10, 2015.

16. Simoes Pires EN, Frozza RL, Hoppe JB, Menezes Bde M and Salbego CG: Berberine was neuroprotective against an in vitro model of brain ischemia: Survival and apoptosis pathways involved. Brain Res 1557: 26-33, 2014.

17. Visnagri A, Kandhare AD and Bodhankar SL: Renoprotective effect of berberine via intonation on apoptosis and mitochondrial-dependent pathway in renal ischemia reperfusion-induced mutilation. Ren Fail 37: 482-493, 2015.

18. Chen K, Li G, Geng F, Zhang Z, Li J, Yang M, Dong L and Gao F: Berberine reduces ischemia/reperfusion-induced myocardial apoptosis via activating AMPK and PI3K-Akt signaling in diabetic rats. Apoptosis 19: 946-957, 2014.

19. Mizushima N and Levine B: Autophagy in mammalian development and differentiation. Nat Cell Biol 12: 823-830, 2010.

20. Deretic V, Saitoh T and Akira S: Autophagy in infection, inflammation and immunity. Nat Rev Immunol 13: 722-737, 2013.

21. Rabinowitz JD and White E: Autophagy and metabolism. Science 330: 1344-1348, 2010.

22. Yan L, Vatner DE, Kim SJ, Ge H, Masurekar M, Massover WH, Yang G, Matsui Y, Sadoshima J and Vatner SF: Autophagy in chronically ischemic myocardium. Proc Natl Acad Sci USA 102: 13807-13812, 2005.

23. Carloni S, Buonocore G and Balduini W: Protective role of autophagy in neonatal hypoxia-ischemia induced brain injury. Neurobiol Dis 32: 329-339, 2008.

24. Jiang M, Liu K, Luo J and Dong Z: Autophagy is a renoprotective mechanism during in vitro hypoxia and in vivo ischemia-reperfusion injury. Am J Pathol 176: 1181-1192, 2010.

25. Hamacher-Brady A, Brady NR and Gottlieb RA: Enhancing macroautophagy protects against ischemia/reperfusion injury in cardiac myocytes. J Biol Chem 281: 29776-29787, 2006.

26. Dosenko VE, Nagibin VS, Tumanovska LV and Moibenko AA: Protective effect of autophagy in anoxia-reoxygenation of isolated cardiomyocyte? Autophagy 2: 305-306, 2006.

27. Nishida K, Yamaguchi O and Otsu K: Crosstalk between autophagy and apoptosis in heart disease. Circ Res 103: 343-351, 2008.

28. Ma X, Liu H, Foyil SR, Godar RJ, Weinheimer CJ, Hill JA and Diwan A: Impaired autophagosome clearance contributes to cardiomyocyte death in ischemia/reperfusion injury. Circulation 125: 3170-3181, 2012.

29. Aki T, Yamaguchi K, Fujimiya $T$ and Mizukami Y: Phosphoinositide 3-kinase accelerates autophagic cell death during glucose deprivation in the rat cardiomyocyte-derived cell line H9c2. Oncogene 22: 8529-8535, 2003.

30. Wang K, Yin XM, Chao DT, Milliman CL and Korsmeyer SJ: BID: A novel BH3 domain-only death agonist. Genes Dev 10: 2859-2869, 1996 
31. Regula KM, Ens K and Kirshenbaum LA: Inducible expression of BNIP3 provokes mitochondrial defects and hypoxia-mediated cell death of ventricular myocytes. Circ Res 91: 226-231, 2002.

32. Bruick RK: Expression of the gene encoding the proapoptotic Nip3 protein is induced by hypoxia. Proc Natl Acad Sci USA 97: 9082-9087, 2000.
33. Kubasiak LA, Hernandez OM, Bishopric NH and Webster KA: Hypoxia and acidosis activate cardiac myocyte death through the Bcl-2 family protein BNIP3. Proc Natl Acad Sci USA 99: 12825-12830, 2002.

34. Wang EY, Gang H, Aviv Y, Dhingra R, Margulets V and Kirshenbaum LA: p53 mediates autophagy and cell death by a mechanism contingent on Bnip3. Hypertension 62: 70-77, 2013. 